\title{
Клинико-функциональный статус, качество жизни и их динамика у больных хронической обструктивной болезнью легких в процессе 6-месячной терапии тиотропия бромидом
}

\author{
ГОУ ДПО Иркутский государственный институт усовершенствования врачей: 664079, Иркутск, м-н Юбилейный, 100
}

\author{
B.A.Chernyak, I.N.Trofimenko
}

\section{Clinical and functional status, quality of life and their changes in COPD patients during 6-month therapy with tiotropium}

\begin{abstract}
Summary
The trial was aimed at investigation of relationships between clinical and functional disorders and quality of life (QoL) in patients with chronic obstructive pulmonary disease (COPD) and effects of tiotropium on these variables. Symptoms, parameters of spirometry and body plethysmography, exercise tolerance, and QoL were analyzed in 138 outpatients with COPD of different severity. The analysis showed a significant effect of lung hyperinflation on dyspnea intensity greatly contributing to QoL and exercise tolerance. Six-month therapy with tiotropium in severe COPD patients significantly reduced the intensity of dyspnea and lung hyperinflation and improved exercise tolerance and QoL. Thus, the reduction in lung hyperinflation is of great importance for improvement of clinical and functional status and QoL in COPD patients.

Key words: chronic obstructive pulmonary disease, lung hyperinflation, quality of life, tiotropium.
\end{abstract}

\section{Резюме}

Целью исследования явилось изучение взаимосвязи клинико-функциональных нарушений, качества жизни (КЖ) и влияние на них терапии тиотропия бромидом (ТБ) у больных хронической обструктивной болезнью легких (ХОБЛ). У 138 пациентов с ХОБЛ различной степени тяжести проанализированы респираторные симптомы, параметры спиро- и бодиплетизмографии, толерантность к физической нагрузке и КЖ. Результаты показали значительное влияние гиперинфляции легких на выраженность одышки, доминирующей в снижении КЖ и толерантности к физическим нагрузкам. Применение ТБ в течение 6 мес. при тяжелой ХОБЛ достоверно уменьшает выраженность одышки и гиперинфляции легких, повышая физическую толерантность и КЖ. Таким образом, уменьшение легочной гиперинфляции является наиболее важным фактором улучшения клинико-функционального статуса и КЖ у больных ХОБЛ.

Ключевые слова: хроническая обструктивная болезнь легких, гиперинфляция легких, качество жизни, тиотропия бромид.

Прогрессирующее и необратимое снижение легочной функции является кардинальным признаком естественного течения хронической обструктивной болезни легких (ХОБЛ). Показатель объема форсированного выдоха за 1-ю с $\left(\mathrm{OФB}_{1}\right)$, являющийся важной функциональной характеристикой обструктивных нарушений и темпов их прогрессирования, остается до настоящего времени определяющим предиктором степени тяжести ХОБЛ (GOLD, 2009) [1]. Вместе с тем ОФВ ${ }_{1}$ как маркер выраженности бронхиальной обструкции не в полной мере соответствует степени тяжести клинических проявлений ХОБЛ, нарушению толерантности к физической нагрузке и снижению качества жизни (КЖ) пациентов [2-5]. В частности, одышка, являясь основной жалобой больных ХОБЛ, имеет низкую коррелятивную связь с ОФВ ${ }_{1}$, особенно, когда значение последнего составляет $<1$ л $[6,7]$.

Тем не менее именно одышка, являясь доминирующим фактором нарушения функционального статуса при данной патологии, заставляет пациента обратиться за медицинской помощью, чаще всего на стадии выраженных вентиляционных нарушений, когда пока- затели ОФВ 1 находятся в пределах 1-1,5 л [8]. Это связано с тем обстоятельством, что выраженность одышки определяется не только степенью бронхиальной обструкции, но и в значительной мере показателями гиперинфляции, которые имеют наибольшую корреляционную связь с тяжестью данного симптома. Так, показано, что при физической нагрузке интенсивность одышки по шкале Борга наиболее высоко коррелирует с инспираторной емкостью $(r=0,69 ; p<0,05)$, а не с ОФВ ${ }_{1}[9]$.

В ряде исследований показано, что терапия тиотропия бромидом (ТБ) существенно уменьшает выраженность респираторного дискомфорта у больных ХОБЛ без значительных изменений показателей бронхиальной обструкции, но при этом сопровождается выраженной динамикой показателей гиперинфляции [10-15]. Именно с этим механизмом связано уменьшение одышки и улучшение толерантности к физической нагрузке при применении бронходилататоров у больных ХОБЛ при отсутствии изменений ОФВ 1 [16].

В связи со сказанным выше целью нашего исследования явилось изучение взаимосвязи клинико- 
функционального статуса и КЖ у больных ХОБЛ в процессе терапии ТБ.

\section{Материалы и методы}

В исследование были включены 138 амбулаторных больных ХОБЛ (125 мужчин и 13 женщин) в возрасте 41-76 лет, которые в соответствии со степенью тяжести заболевания были распределены на 4 группы: 1 -я группа - легкая степень ХОБЛ $(n=21) ; 2$-я среднетяжелая $(n=36)$; 3-я - тяжелая $(n=58)$; 4-я крайне тяжелая степень ХОБЛ ( $n=23)$. Диагностика ХОБЛ и оценка степени ее тяжести осуществлялись на основании критериев GOLD [1]. Интенсивность респираторных симптомов оценивалась с использованием стандартизованных шкал. Симптомы кашля и продукции мокроты анализировались по 3-балльной шкале Paggiaro, одышка - по 4-балльной шкале MRC. Оценка показателей легочной функции проводилась на основании результатов спирометрии и бодиплетизмографии. Для исследования толерантности к физической нагрузке проводился 6-минутный шаговый тест (6-МШТ) в соответствии со стандартным протоколом, который включал в себя оценку пройденного расстояния в метрах, выраженность одышки в конце теста по 10-балльной аналогово-визуальной шкале Борга (0 - нет одышки, 10 максимальная одышка). В качестве интегрального показателя клинико-функционального статуса больных ХОБЛ проанализирован bode-индекс [17], который представляет собой суммарную оценку 4 параметров: 1) В - ВМІ - индекс массы тела (ИМТ), определяемый по формуле: масса тела (кг) / рост $\left(\mathrm{M}^{2}\right)$; 2) O - obstruction - выраженность бронхиальной обструкции по показателю ОФВ 1 (\% от должного значения); 3) D - dyspnea - одышка по шкале MRC; 4) $\mathrm{E}$ - exercise tolerance - толерантность к физической нагрузке с оценкой пройденного расстояния в метрах (6-МШТ). Bode-индекс характеризовался 3 градациями: низкий индекс $-0-3$ балла; средний $-4-6$ баллов; высокий $-7-10$ баллов. Уровень индекса, по данным его авторов, отражает степень клинико-функциональных нарушений у больных ХОБЛ, при этом, чем выше его значение, тем в большей степени выражены функциональные нарушения. Изучение специфического КЖ проведено с помощью специализированного респираторного вопросника Госпиталя св. Георгия (St. George's Respiratory Questionnaire - SGRQ). Анализ показателей КЖ проводился по каждому домену вопросника: "Симптомы", "Активность", "Влияние" и "Общее КЖ".

Влияние ТБ на клинико-функциональный статус и КЖ оценивалось у 40 больных из группы тяжелой ХОБЛ (все мужчины в возрасте 43-69 лет; средний возраст - 57,9 $\pm 8,7$ лет). После 2-недельного вводного периода больные были рандомизированы в 2 равные группы: 20 больным был назначен ТБ (Спирива) в дозе 18 мкг в сутки через "Хандихалер", 20 больным ТБ не назначался (контрольная группа). Лечебный период продолжался 6 мес.: в контрольной группе пациенты принимали только коротко- действующие бронходилататоры (КДБД) (Атровент, Вентолин, Сальбутамол, Беротек, Беродуал), назначенные им до рандомизации, в группе пациентов, получающих лечение ТБ, использовался Вентолин или Сальбутамол в режиме “по требованию”. Клинико-функциональные показатели и параметры КЖ оценивались в сравниваемых группах исходно и через 6 мес. терапии. Статистическая обработка результатов проводилась с помощью общепринятых в медицине методов вариационной статистики, корреляционного и множественного регрессионного анализов. Сравнительный анализ выполнен с использованием критерия Манна-Уитни.

\section{Результаты и обсуждение}

Полученные результаты показали достоверное усиление одышки по мере нарастания тяжести ХОБЛ. Вместе с тем индивидуальный анализ этого показателя в пределах одной стадии заболевания свидетельствует о значительных колебаниях выраженности одышки. Так, в группе больных тяжелой ХОБЛ (3-я стадия) индивидуальные показатели одышки по шкале MRC значительно варьировались: от легкой $(13,8 \%)$, умеренной и тяжелой $(77,6 \%)$ до очень тяжелой у $8,6 \%$ больных. Отмеченная гетерогенность одышки в рамках одной стадии ХОБЛ характеризуется наиболее высокими корреляционными связями между степенью диспноэ и параметрами легочной гиперинфляции: для остаточного объема легких (ООЛ) и инспираторной емкости (Евд.) $(r=0,73$ и $-0,54$ соответственно; $p<0,05)$, тогда как коэффициент корреляции для степени одышки и ОФВ 1 был значительно ниже.

Для раздельной оценки вклада бронхиальной обструкции и легочной гиперинфляции в формирование одышки при ХОБЛ нами проведен множественный регрессионный анализ показателей функции внешнего дыхания (ФВД). Полученные высокие значения коэффициентов множественной корреляции $(R)$ и детерминации $\left(\mathrm{R}^{2}\right)$ между степенью одышки и показателями ФВД (0,91 и 0,84 соответственно; $p<0,0001)$ подтвердили, что степень одышки в значительной степени зависит не только от бронхиальной обструкции, но и от уровня гиперинфляции. Об этом свидетельствуют значения $\beta$-коэффициентов стандартизированных коэффициентов регресса, величина которых позволяет сравнивать относительный вклад каждой независимой переменной в предсказание зависимой переменной. В частности, для ООЛ и Евд. $\beta$-коэффициент равен 0,41 и $-0,40$, для $\mathrm{OФB}_{1}$ и максимальной объемной скорости потока при $75 \%$ выдохнутого легочного объема $\left(\mathrm{MOC}_{75}\right)$ $-0,56$ и 0,36 соответственно ( $p<0,001-$ во всех случаях). Таким образом, результаты регрессионного анализа показали, что в генезе одышки вклад гиперинфляции имеет значение, сопоставимое с бронхиальной обструкцией, чем, вероятно, и обусловлены значительные индивидуальные колебания выраженности этого симптома у больных ХОБЛ в пределах одной стадии заболевания. 
Другим важным индикатором состояния больного, помимо одышки, является толерантность к физической нагрузке. Результаты 6-МШТ показали снижение физических возможностей параллельно с нарастанием степени тяжести заболевания. Так, пройденное расстояние уменьшалось с $484 \pm 39$ м при I стадии до $289 \pm 62$ м при IV стадии ХОБЛ, а интенсивность одышки в конце 6-МШТ, оцененная по шкале Борга, увеличивалась с $1,8 \pm 0,7$ до $5,7 \pm 1,7$ соответственно. Однако индивидуальный анализ этих же параметров в пределах одной стадии заболевания демонстрирует, как и при оценке одышки, широкий диапазон колебаний. В качестве интегрального показателя клинико-функционального статуса больных ХОБЛ нами проанализирован bodeиндекс в зависимости от степени тяжести заболевания (рисунок). Значение медианы bode-индекса закономерно увеличивается с 0 до 7 баллов по мере прогрессирования заболевания $(p<0,001)$. Индивидуальный анализ индекса в пределах одной стадии заболевания показал, что 100 \% больных легкой ХОБЛ соответствовали низкому bode-индексу (0-3 балла), и напротив, $100 \%$ больных крайне тяжелой ХОБЛ - высокому индексу (7-10 баллов). Больные среднетяжелой ХОБЛ в большинстве случаев $(88,9 \%)$ имели низкий и средний (в $11,1 \%$ случаев) bode-индекс (4-6 баллов). Вместе с тем bode-индекс у больных тяжелой ХОБЛ характеризовался значительной вариабельностью: 13,8 \% больных имели высокий индекс (7-10 баллов), 53,4 \% - средний (4-6 баллов), $32,8 \%$ больных тяжелой ХОБЛ - низкий bode-индекс (0-3 балла). Проведенный анализ показывает, что примерно треть пациентов с ОФВ 1 , соответствующим тяжелой ХОБЛ по критериям GOLD, имеют низкие значения bode-индекса ( $0-3$ балла), за счет достаточно хорошей толерантности к физической нагрузке и незначительного уровня одышки. Таким образом, анализ bode-индекса подтверждает значительную гетерогенность группы больных тяжелой ХОБЛ, что было показано в отношении одышки и толерантности к физической нагрузке, а показатели бронхиальной обструкции $\left(\mathrm{OФB}_{1}\right)$ не являются

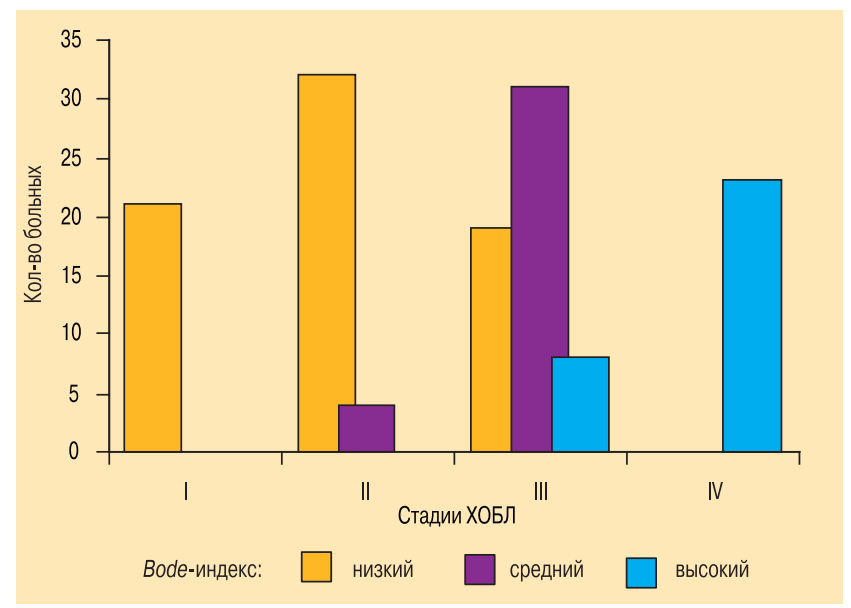

Рисунок. Распределение больных ХОБЛ в зависимости от значения bode-индекса

высокозначимыми предикторами выраженности одышки и нарушения толерантности к физической нагрузке у больных ХОБЛ.

КЖ по всем доменам вопросника ухудшалось по мере утяжеления ХОБЛ, демонстрируя закономерную связь между уровнем снижения КЖ и степенью тяжести ХОБЛ. При анализе взаимоотношений показателей КЖ и параметров клинико-функционального статуса больных ХОБЛ для большинства из них отмечена существенная корреляционная взаимосвязь (табл. 1).

Наибольшее влияние на КЖ оказывает уровень одышки: наибольшее значение коэффициента корреляции определяется для таких доменов, как "Активность" $(r=0,83, p<0,05)$ и "Общее КЖ" $(r=0,81$, $p<0,05)$ шкалы MRC, и несколько ниже - корреляциионные связи между этими доменами и одышкой по шкале Борга ( $r=0,70$ и 0,73 соответственно, $p<0,05)$. В отличие от одышки, другие симптомы ХОБЛ - кашель, продукция мокроты - характеризовались низким уровнем корреляционных связей. Из анализируемых параметров ФВД наиболее тесная связь с КЖ отмечается для ОФВ 1 и ООЛ. Взаимосвязь между КЖ и клинико-функциональными параметрами, характеризующими уровень одышки

Таблица 1

Взаимосвязь между клинико-функциональныл статусом и КЖу больных ХОБЛ

\begin{tabular}{|c|c|c|c|c|}
\hline Клинико-функциональные показатели & "Симптомы" & "Активность" & "Влияние" & "Общее КЖ" \\
\hline Одышка (MRC) & 0,64 & 0,83 & 0,74 & 0,81 \\
\hline Кашель & 0,51 & 0,27 & 0,40 & 0,41 \\
\hline Продукция мокроты & 0,26 & $0,10^{*}$ & $0,12^{*}$ & $0,15^{*}$ \\
\hline Длительность заболевания & 0,53 & 0,40 & 0,50 & 0,51 \\
\hline ИК, пачко-лет & $-0,06^{*}$ & $-0,22$ & $-0,23$ & $-0,21$ \\
\hline Расстояние в 6-МШт & $-0,45$ & $-0,65$ & $-0,59$ & $-0,63$ \\
\hline Одышка по шкале Борга & 0,68 & 0,70 & 0,65 & 0,73 \\
\hline ФЖЕЛ & $-0,12^{*}$ & $-0,30$ & $-0,26^{*}$ & $-0,29 *$ \\
\hline $0 \Phi B_{1}$ & $-0,45$ & $-0,65$ & $-0,57$ & $-0,68$ \\
\hline Евд. & $-0,16^{*}$ & $-0,40$ & $-0,34$ & $-0,39$ \\
\hline оол & 0,63 & 0,47 & 0,54 & 0,62 \\
\hline R tot & 0,31 & 0,36 & 0,33 & 0,39 \\
\hline
\end{tabular}

Примечание: * - p > 0,05; для остальных значений $r$ - p<0,05; ФЖЕЛ - форсированная жизненная емкость легких; R tot - сопротивление дыхательных путей. 
Динамика выраженности симптомов ХОБЛ $(M \pm m)$

\begin{tabular}{|c|c|c|c|c|c|c|}
\hline Симптомы & Группы & Исходно & $p_{1}$ & Через 6 мес. & $p_{1}$ & $p_{2}$ \\
\hline \multirow[t]{2}{*}{ Одышка } & 1-я группа & $3,3 \pm 0,7$ & $>0,05$ & $2,6 \pm 0,5$ & $<0,001$ & $<0,001$ \\
\hline & 2-я группа & $3,2 \pm 0,6$ & & $3,3 \pm 0,7$ & & $>0,5$ \\
\hline \multirow[t]{2}{*}{ Кашель } & 1-я группа & $1,8 \pm 0,6$ & $>0,05$ & $1,0 \pm 0,0$ & $<0,001$ & $<0,001$ \\
\hline & 2-я группа & $2,1 \pm 0,6$ & & $2,0 \pm 0,0$ & & $>0,5$ \\
\hline \multirow[t]{2}{*}{ Мокрота } & 1-я группа & $1,3 \pm 0,5$ & $>0,05$ & $1,0 \pm 0,0$ & $<0,001$ & $<0,05$ \\
\hline & 2-я группа & $1,1 \pm 0,3$ & & $1,3 \pm 0,5$ & & $>0,05$ \\
\hline
\end{tabular}

Примечание: $p_{1}$ - при сравнении между группами; $p_{2}$ - при сравнении с исходными показателями.

и вентиляционных нарушений, хорошо корреспондирует с толерантностью к физической нагрузке. Получена значимая обратная зависимость между всеми доменами вопросника и пройденной больными дистанцией в 6-МШТ (табл. 1). Наиболее высокий коэффициент корреляции отмечен для домена "Активность" ( $r=-0,65, p<0,05)$, характеризующего влияние ХОБЛ на физическую активность, что подтверждает специфичность этого параметра.

Через 6 мес. терапии ТБ у больных тяжелой ХОБЛ отмечалось достоверное уменьшение уровня одышки и клинических проявлений бронхитического синдрома по сравнению как с исходными показателями, так и с контрольной группой (табл. 2).

Разница показателей одышки, кашля и продукции мокроты между больными, принимающими ТБ, и группой сравнения составила 0,$7 ; 1,0$ и 0,3 балла соответственно $(p<0,05)$. Потребность в КДБД на фоне приема ТБ значительно уменьшилась (с 3,6 до $2,4$ раза в сутки; $p<0,001)$, тогда как в группе сравнения отмечена тенденция к увеличению использования КДБД "по потребности".

Уменьшение выраженности одышки в процессе лечения ТБ сопровождалось значительной динамикой показателей ФВД, характеризующих уровень гиперинфляции легких (табл. 3). Так, динамика средних значений ООЛ и Евд. к 6 мес. лечения ТБ составила 430 мл и 330 мл соответственно $(p<0,05)$.

Менее показательными были изменения параметров бронхиальной обструкции. К окончанию лечебного периода отмечено достоверное увеличение $\mathrm{OФB}_{1}$ (до применения бронходилататора) в группе ТБ по сравнению с исходными показателями на 120 мл $(p=0,006)$. Однако статистически значимых различий к 6 мес. терапии между постбронходилатационными показателями и исходными величинами, а также группой сравнения, не достигнуто. С положительной динамикой симптомов заболевания и параметров гиперинфляции корреспондирует увеличение толерантности к физической нагрузке. Через 6 мес. лечения ТБ пройденное расстояние в 6-МШТ возросло на 37 м, а одышка по шкале Борга уменьшилась на 1 балл относительно исходных показателей $(p<0,01)$. В группе сравнения показатели толерантности к физической нагрузке существенно не изменились.

Анализ параметров КЖ показал достоверное улучшение в группе ТБ по домену "Симптомы" и общему показателю к окончанию лечебного периода с 72,1 до 57,6 баллов $(p<0,001)$ и с 55,1 до 49,9 баллов $(p<0,05)$ соответственно. Улучшение отмечалось как в сравнении с исходными параметрами КЖ, так и показателями КЖ в группе КДБД $(p<0,05)$. По другим доменам отмечена тенденция к улучшению показателей КЖ, однако статистически значимой динамики показателей в процессе лечения не прослеживалось. В группе сравнения отмечено достоверное ухудшение КЖ по домену "Влияние" с 40,5 до 46,1 баллов $(p<0,05)$ за 6-месячный период, показатели других доменов вопросника имели также тенденцию к отрицательной динамике.

\section{Заключение}

По мере прогрессирования ХОБЛ нарастает степень клинико-функциональных нарушений и ухудшается КЖ больных. Одышка является доминирующим фактором нарушения функционального статуса,

Таблица 3

Динамика показателей ФВД $(M \pm m)$

\begin{tabular}{|c|c|c|c|c|c|c|}
\hline Симптомы & Группы & Исходно & $p_{1}$ & Через 6 мес. & $p_{1}$ & $p_{2}$ \\
\hline ОФВ ${ }_{1}$ до бронхо- & 1-я группа & $38,4 \pm 7,9$ & $>0,05$ & $41,3 \pm 5,8$ & $>0,05$ & $>0,05$ \\
\hline литика, \% оолж. & 2-я группа & $40,2 \pm 5,7$ & & $43,2 \pm 4,6$ & & $>0,05$ \\
\hline ОФВ 1 после бронхо- & 1-я группа & $41,9 \pm 6,2$ & $>0,05$ & $43,1 \pm 5,4$ & $>0,05$ & $>0,05$ \\
\hline литика, \% & 2-я группа & $42,8 \pm 7,8$ & & $42,3 \pm 4,0$ & & $>0,5$ \\
\hline \multirow[t]{2}{*}{ ООЛ, \% } & 1-я группа & $211,7 \pm 29,9$ & $>0,05$ & $193,3 \pm 31,8$ & $>0,05$ & $<0,001$ \\
\hline & 2-я группа & $205,8 \pm 30,1$ & & $201,9 \pm 35,6$ & & $>0,05$ \\
\hline \multirow[t]{2}{*}{ Евд., л } & 1-я группа & $2,3 \pm 0,8$ & $>0,05$ & $2,63 \pm 0,60$ & $>0,05$ & $<0,05$ \\
\hline & 2-я группа & $2,4 \pm 0,6$ & & $2,4 \pm 0,5$ & & $>0,5$ \\
\hline
\end{tabular}

Примечание: $p_{1}$ - при сравнении между группами; $p_{2}$ - при сравнении с исходными показателями. 
снижения толерантности к физическим нагрузкам и КЖ у больных ХОБЛ. В пределах одной стадии заболевания показатели клинико-функционального статуса и параметры КЖ могут существенно варьироваться. Это подтверждает гетерогенность тяжелой ХОБЛ не только по клинико-функциональным характеристикам, но и по показателям КЖ, отражающим субъективное восприятие заболевания. Полученные результаты свидетельствуют о том, что наряду с выраженностью бронхиальной обструкции существенный вклад в нарушение функционального статуса вносит легочная гиперинфляция.

Шестимесячная терапия больных тяжелой ХОБЛ сопровождается уменьшением симптомов заболевания, в первую очередь одышки, и повышением толерантности к физической нагрузке, что обусловливает, в конечном счете, значительное улучшение КЖ. Динамика параметров ФВД в процессе лечения свидетельствует о том, что положительный клиникофункциональный эффект ТБ и улучшение КЖ при тяжелой ХОБЛ в значительной мере обусловлены снижением легочной гиперинфляции. Выраженность последней необходимо учитывать при оценке степени тяжести ХОБЛ и эффективности ее лечения.

\section{Литература}

1. Global strategy for the diagnosis, management, and prevention of chronic obstructive pulmonary disease (2009). www.goldcopd.org

2. Стандарты по диагностике и лечению больных хронической обструктивной болезнью легких (ATS / ERS, пересмотр 2004). Пер. с англ. под ред. Чучалина А.Г. М.: Атмосфера; 2005.

3. Ferguson G.T. The ins and outs of breathing: an overview of lung mechanics. Eur. Respir. Rev. 2004; 13 (89): 30-34.

4. MacNee W., ZuWallack R.L., Keenan J. Clinical management of chronic obstructive pulmonary disease. USA: Professional Communications, 2005.

5. O'Donnell D.E. Lung mechanics in COPD: the role of tiotropium. Eur. Respir. Rev. 2004; 13 (89): 40-44.

6. Ambrosino $N$. Dyspnoea and its measurement. Breathe 2004; 1 (2): 101-107.
7. Mahler D.A. Understanding dyspnoea in COPD: mechanisms, measurement and management. Eur. Respir. Rev. 2002; 12 (82): 25.

8. Agusti A.G.N. The science of symptoms in chronic obstructive pulmonary disease. Eur. Respir. Rev.1999; 9 (67): $160-164$.

9. Boni E., Corda L., Franchini D. et al. Volume effect and exertional dyspnoea after bronchodilator in COPD patients with and without expiratory flow limitation at rest. Thorax 2002; 57: 528-532.

10. Celli B.R., ZuWallak R.L., Wang S. et al. Improvement in resting inspiratory capacity and hyperinflation with tiotropium in COPD patients with increased static lung volumes. Chest 2003; 124: 1743-1748.

11. Ferguson G.T. Why Does the Lung Hyperinflate. The Proceedings of the American Thoracic Society 2006; 3: 176-179.

12. Marco F.Di., Milic-Emili J., Boveri B. et al. Effect of inhaled bronchodilators on inspiratory capacity and dyspnoea at rest in COPD. Eur. Respir. J. 2003; 21: 86-94.

13. Gelb A.F., Taylor C.F., McClean P.A. et al. Tiotropium and simplified detection of dynamic hyperinflation. Chest 2007; 131: 690-695.

14. Verkindre C., Bart F., Aguilaniu B. et al. The effect of tiotropium on hyperinflation and exercise capacity in chronic obstructive pulmonary disease. Respiration 2006; 73 (4): 420-427.

15. O'Donnell D.E., Fluge T., Gerken F. et al. Effects of tiotropium on lung hyperinflation, dyspnoea and exercise tolerance in COPD. Eur. Respir. J. 2004; 23: 832-840.

16. O'Donnell, D.E. Exercise and dyspnoea. Eur. Respir. Rev. 2002; 12 (82): 36-39.

17. Celli B.R., Cote C.G., Marin J.M. et al. The body-mass index, airflow obstruction, dyspnea, and exercise capacity index in chronic obstructive pulmonary disease. N. Engl. J. Med. 2004; 350: 1005-1012.

\section{Информация об авторах:}

Черняк Борис Анатольевич - д. м. н., проф., зав. кафедрой аллергологии и пульмонологии Иркутского ГИУВ; тел.: 8-902-568-78-70; e-mail: bores@angara.ru

Трофименко Ирина Николаевна - к. м. н., ассистент кафедры аллергологии и пульмонологии Иркутского ГИУВ; тел.: 8-914-877-80-96, e-mail: tin11@mail.ru 


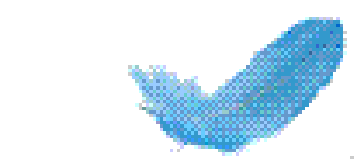

UPLIFT:



СПИРИВА॰ продемонстрировапа

долговрочное попожительное виияние

на клиническое төчение XОБЛ, по данныи

4x nemero rno6aniнoro nсепедования UPLIFT²:

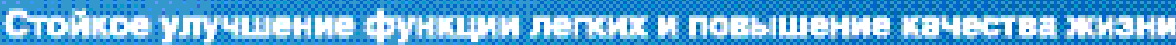
Снимение риска обострений и связаннькх с ними госпитализачий Влияние на смертность

\section{Назначайте СПИРИВУ раньше и nomoraйте вачим пациентам сохранить боnеe активный о6раз жизни'х3}

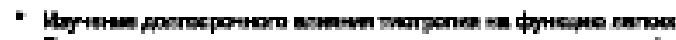



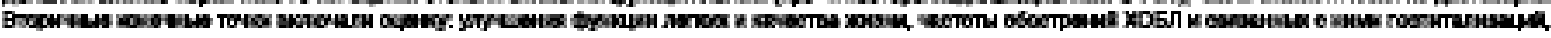

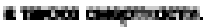

neveperype:

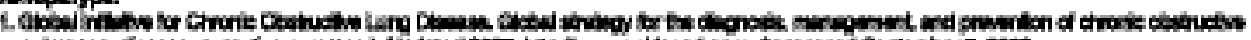

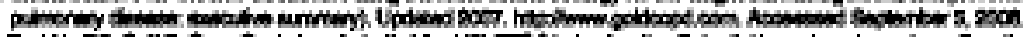





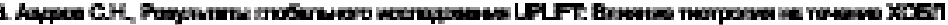

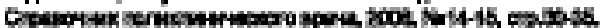

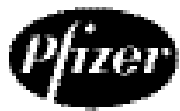



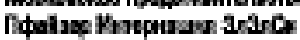

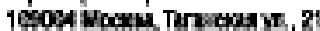
Тยн中:н (C25) 85e-5585

\section{(1) Boehringer}


Becwerap Atrazunfu Gapua fidy

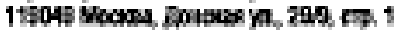
Teropar [eq) ets-rsor

\section{SPIRIVA}

(tiotropium)

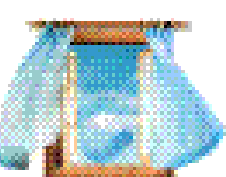

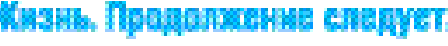

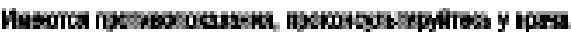

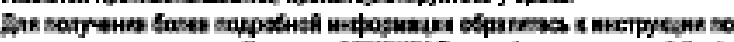

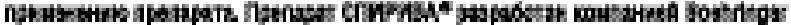



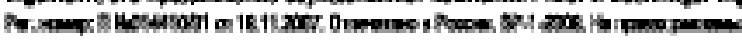

